\title{
REPRESENTAÇÕES SOCIAIS DE ESTUDANTES E DE SEUS RESPONSÁVEIS SOBRE A CIÊNCIA NO DIA A DIA - OU CIÊNCIA INFORMAL
}

\author{
SOCIAL REPRESENTATIONS OF STUDENTS AND THEIR GUARDIANS \\ ABOUT DAY-TO-DAY SCIENCE - OR INFORMAL SCIENCE
}

\author{
EDUARDO MOZART MACHADO1 \\ MARIANA A. DE ANDRADE BOLOGNA SOARES ${ }^{2}$
}

\section{RESUMO}

A motivação inicial para o desenvolvimento desta pesquisa era entender o contexto em que as famílias se encontram em relação à educação de seus filhos, questionando quais as possíveis influências da Escola e da Família na construção de Representações Sociais sobre Ciência Informal por estudantes de Ensino Fundamental e tendo como objetivo analisar as Representações Sociais de estudantes e de seus responsáveis sobre a ciência no dia a dia. A partir da aplicação da análise prototípica, instrumento de coleta consolidado no campo das Representações sociais, foi possível observar que as famílias têm pouca influência na construção das Representações Sociais de seus filhos, sendo a Escola a principal referência de Ciência destes indivíduos e, portanto, suas Representações estão relacionadas ao ambiente escolar, suas atividades e métodos, proporcionando um distanciamento da Ciência no cotidiano destes jovens.

Palavras-chave: Família. Educação. Escola.Psicologia Social.

\section{ABSTRACT}

The initial motivation for the development of this research was to understand the context in which families find themselves in relation to their children's education, questioning what are the possible influences of the School and the Family in the construction of Social Representations about Informal Science by Elementary School students and aiming to analyze the Social Representations of students and their guardians about science in their daily lives. From the application of prototypical analysis, a consolidated collection instrument in the field of Social Representations, it was possible to observe that families have little influence on the construction of their children's Social Representations, with the School being the main Science reference of these individuals and, therefore, their Representations are related to the school environment, their activities and methods, providing a distance from Science in the daily lives of these young people.

Keywords: Family. Education. School. Social Psychology.

\footnotetext{
1 Mestre em Ensino de Ciências e Educação Matemática pela Universidade Estadual de Londrina (UEL). Professor de Ciências e Biologia pela Secretaria de Estado da Educação do Paraná (SEED), Paranaguá, Paraná. Email: eduardomozart@seed.pr.gov.br. ORCID: https://orcid.org/ 0000-0003-1707-9176.

2 Doutora em Educação para a Ciência pela Universidade Estadual Paulista Júlio de Mesquita Filho, (UNESP). Professora associada da Universidade Estadual de Londrina (UEL), Londrina, Paraná. Email: mariana.bologna@gmail.com. ORCID: https://orcid.org/0000-0002-1945-4606.
} 


\section{INTRODUÇÃO}

0 interesse para a realização deste estudo surgiu da motivação em entender 0 contexto em que as famílias se encontram em relação à educação escolar de seus filhos, inicialmente pensando na relação Família x Escola, procurando questionar: "como os responsáveis educam e acompanham seus filhos na realização das atividades escolares?".

Neste sentido, surgiram novas indagações buscando um problema de pesquisa que abarcasse a relação Família x Escola, as Representações Sociais e o Ensino de Ciências. Sendo assim, o questionamento que se sucedeu e guiou esta pesquisa foi: "Quais as possíveis influências da Escola e da Família na construção de Representações Sociais sobre Ciência Informal por estudantes de Ensino Fundamental?".

Por este caminho, buscando um referencial teórico que sustentasse estas ideias e que permitisse aproximar o questionamento inicial com o Ensino de Ciências, foi no conceito de Representações Sociais (CABECINHAS, 2003; MOSCOVICI, 2003; CASTR0, 2004) que se encontrou a fundamentação teórica para guiar a pesquisa aqui desenvolvida.

Assim sendo, 0 objetivo desta pesquisa foi analisar as Representações Sociais de estudantes e de seus responsáveis sobre a ciência no dia a dia - ou Ciência Informal - e qual a influência da família e da escola nas Representações destes estudantes.

\section{EDUCAÇÃO CIENTÍFICA FORMAL, INFORMAL E A NATUREZA DA CIÊNCIA}

0 conhecimento está presente em todos os espaços onde há vida humana, não somente nas escolas, mas também na família, em igrejas, sindicatos, praças, associações de moradores, parques botânicos, museus, entre tantos outros locais ou momentos que podem ser mencionados (MIRLENO; LEITE, 2014). Segundo os mesmos autores, "com a promulgação da Lei de Diretrizes e Bases da Educação Nacional (LDBEN No 9394/96) a escola formal deixou de ser um espaço hegemônico de educação e de formação humana" (p. 5874, 2014).

Assim, nota-se uma Educação Científica Formal, reconhecida oficialmente por órgãos e instituições, que tem por objetivo oferecê-la na forma de cursos com diferentes níveis, graus, programas, currículos e diplomas em escolas, universidades e outras instituições reconhecidas oficialmente por entidades governamentais (GASPAR, 2002).

Por outro lado, devido à complexidade das interações sociais humanas, conforme apontam Cascais e Terán (2011), existe uma Educação Científica Informal, que se diferencia por não possuir métodos e estrutura definida, acontecendo em qualquer espaço de convivência humana e não obedecendo nenhum parâmetro estruturante anterior. Para que aconteça a Educação Informal, basta existirem pessoas interessadas em aprender e pessoas interessadas em ensinar qualquer tipo de conhecimento (GASPAR, 2002).

Existe ainda a Educação Científica Não-Formal que, para Vieira (2005), pode ser definida como uma educação que possui um direcionamento, seguindo objetivos, metodologias e conteúdos bem definidos, ocorrendo em locais públicos ou privados como museus, planetários, zoológicos e que obedecem também aos conhecimentos científicos e trabalhados também na Educação Formal.

Sendo assim, nos deparamos então, com três formas de educação científica: a educação formal, a educação informal e a educação não-formal, sendo distinguidas basicamente pelos locais onde ocorrem e pela sistematização que sofrem, podendo ser muito organizadas como a educação 
formal, ou sem nenhuma organização e sistematização, como é o caso da educação informal (GASPAR, 2002; FOUREZ, 2003; VIEIRA, 2005).

\section{A IMPORTÂNCIA DA FAMÍLIA NA CIÊNCIA INFORMAL E EDUCAÇÃO ESCOLAR}

A Educação prepara o ser humano para 0 desenvolvimento de suas atividades ao longo da vida, sendo a Família a entidade social que, certamente, exerce as maiores influências nos indivíduos e é neste ambiente que a maioria das pessoas recebe seus primeiros contatos com o mundo, quase sempre estabelecendo vínculos muito fortes entre seus semelhantes, sejam eles positivos ou negativos (CASCAIS; TERÁN, 2011). De qualquer maneira, é nítida a importância de um núcleo familiar para o desenvolvimento de muitas das carências essenciais humanas, como a espiritualidade, a cognição, a afetividade, o relacionamento social, a educação e a saúde.

Segundo Polônia e Dassen (2005), é inegável a contribuição da família para o desenvolvimento e aprendizagem humana, sendo seus papéis principais a socialização e inclusão dos indivíduos no contexto cultural por meio do ensino da língua materna, dos símbolos e regras sociais, que engloba aspectos da educação informal e auxiliando na educação formal em colaboração com a escola.

Conforme aponta Carvalho (2000), em relação à escola, considera-se como participação dos pais na educação de seus filhos o comparecimento às reuniões, a atenção à comunicação com a equipe pedagógica e o acompanhamento dos deveres de casa e das notas. Por esses motivos é indispensável para profissionais da educação, compreender as relações entre a participação da família no desenvolvimento escolar dos indivíduos e, como salienta Picanço (2012), a escola deve ser promotora de estratégias que aproximem os pais da realidade escolar.

Portanto, é importante uma relação próxima entre a escola e a família, uma vez que a escola é a principal promotora dos conhecimentos científicos nas primeiras fases de vida de uma criança (ALVES-MAZZOTTI, 1994). Além disto, Zancan (2000) também considera que 0 analfabetismo científico aumentará as desigualdades, excluindo do mercado de trabalho as maiorias que hoje já são excluídas.

Tendo em vista a importância do conhecimento científico e da família no processo educacional e de formação dos jovens, concordamos com Moreira (2004) que aponta que o objetivo do Ensino de Ciência é permitir ao aluno o compartilhamento de significados científicos e interpretações científicas da realidade e do mundo e, portanto, segundo Marques (2002) a escola é 0 espaço próprio onde a educação trata do conhecimento científico, com os seus níveis de ensino, suas regras e procedimentos, entretanto, ela não pode mais ater-se somente a esse espaço e faz-se necessário lançar mão de outros ambientes que possam favorecer uma aprendizagem mais significativa e instigante aos estudantes. Sendo assim, deve existir a compreensão por parte da sociedade, das famílias e, principalmente da escola, de seus papéis no desenvolvimento educacional formal dos indivíduos.

\section{REPRESENTAÇÕES SOCIAIS}

Tendo em vista os conceitos de alfabetização científica, os tipos de Ciência anteriormente discutidos, bem como o papel da família na educação básica e científica dos jovens, é concebível um olhar sob a luz da Teoria das Representações Sociais, na busca pela compreensão de como estes sujeitos representam a Ciência no dia a dia, uma vez queestas Representações têm papel crucial na formação da identidade dos indivíduos (VALA, 1997) e na maneira como eles enxergam a realidade 
que os rodeia, bem como as ações que podem tomar em seu cotidiano e ao longo de suas vidas (MOSCOVICl, 2003)

A partir de 1912 Durkheim, em sua obra As formas elementares de vida religiosa, coloca as Representações Coletivas como um conceito importante da análise sociológica (PINHEIRO-FILHO, 2004). Estas Representações são construídas no cotidiano das interações sociais de maneira coletiva, a partir das influências exteriores da sociedade nos sujeitos, podendo, qualquer coisa ou objeto ser representada mentalmente, ou seja, as Representações Coletivas expressam de forma simples o que os humanos pensam sobre si mesmos e sobre a realidade que os cercam (OLIVEIRA, 2012). As Representações Coletivas, assim como as Representações Sociais são, portanto, formas de conhecimento socialmente produzido e construído (MOSCOVICI, 1979; MOSCOVICl, 2003; OLIVEIRA, 2012).

0 conceito de Representação Social é amplo e circula em diversas áreas das Ciências Humanas, ganhando corpo teórico a partir dos estudos e aprofundamentos de Serge Moscovici e Denise Jodelet, passando a servir como ferramenta de investigação para diferentes campos do conhecimento (ARRUDA, 2002). Moscovici suscita o conceito de Representação Social a partir do conceito de Representação Coletiva de Durkheim, por considerar este último um conceito inapropriado para explicar as relações sociais modernas, afirmando que as representações coletivas constituíam-se em uma classe muito generalizada de fenômenos psíquicos e sociais que compreendiam o que chamamos de ciência, ideologia, mito, entre outros, preocupando-se com os fenômenos em si e não com a busca de explicações sobre a origem destes fenômenos (MOSCOVICI, 1979; ALVES-MAZZOTTI, 1994; ARRUDA, 2002). Sendo assim, "para o homem chamado moderno a Representação Social é somente uma das vias para captar o mundo concreto, circunscrita em seus fundamentos e em suas conseqüências (sic)" (MOSCOVICI, 1979, p. 29). Portanto, as Representações Sociais são utilizadas pelas pessoas para dar sentido ao mundo que as cerca, utilizando-se de símbolos, palavras, imagens, de uma forma individual e também coletiva (MOSCOVICI, 2003).

Spink (1993) destaca diversas funções para as Representações Sociais, entre elas uma função social, orientadora das condutas e das comunicações, uma função afetiva, que garante proteção e legitimação das identidades sociais e uma última função cognitiva, que permite a familiarização com a novidade. Dentro destas funções, para compreender a construção das representações sociais é necessário analisar os processos de objetivação e ancoragem que influenciam em sua formação (MARTINEZ et. al., 2012).

Existe uma possibilidade de conciliação entre as teorias das Representações Sociais com a teoria da Identidade Social pois, conforme argumenta Breakwell (1993), a teoria das Representações Sociais é amparada ou pode ser complementada pela teoria da Identidade Social, pois o entendimento da identidade dos sujeitos auxilia em dois aspectos para o entendimento da Representação, primeiro a identidade permite descrever os processos que podem estar em ação na forma de Representação e, em um segundo caso, também permite determinar os processos de familiarização do "novo", ou seja, do objeto que está sendo representado. Além disto, conforme o mesmo autor, um dos problemas das Representações é que ela sozinha não responde de onde surgem e como uma Representação assume sua forma em determinado grupo.

Sendo assim, destaca-se a importância do estudo das Representações Sociais no campo de pesquisa em Educação em Ciências, pois o entendimento das Representações dos participantes acerca do contexto escolar permite, conforme argumenta Alves-Mazzotti (1994, p. 74), "a compreensão do que se passa em classe durante a interação educativa propriamente dita, tanto do ponto de vista dos objetos do conhecimento a ser ensinado quanto dos mecanismos psicossociais em ação no processo educacional". 
Portanto serão discutidos no próximo item, fechando esta sessão teórica, aspectos relacionados ao papel da Família e da Escola na construção das Representações dos estudantes.

\section{REPRESENTAÇÕES SOCIAIS, FAMÍLIA E EDUCAÇÃO CIENTÍFICA}

A maioria das relações sociais próximas, dos objetos produzidos ou consumidos, das comunicações trocadas estão impregnadas por Representações Sociais e é sabido que correspondem, por um lado, à substância simbólica que entra em sua elaboração e, por outro, à prática que produz essa substância, assim como a Ciência ou os mitos correspondem a uma prática científica e mítica (MOSCOVICI, 1979). Selingard et al. (2017, p. 14781). afirmam que "a Representação Social de um grupo pode ser um agente influenciador no processo de aprendizagem dos sujeitos", colocando ainda que, "parte das concepções criadas pelos indivíduos são frutos de uma construção social, e por mais ativo que os sujeitos sejam, eles se submetem as concepções do grupo social que estão inseridos".

Portanto é importante observar que, por viverem em sociedade, os adultos principalmente ligados ao ambiente familiar, são os primeiros a influenciar o processo de aprendizagem das crianças, propiciando os primeiros estímulos para o desenvolvimento integral humano (TAVEIRA-BISPO, 2015) e "é através dos afetos que 0 ser humano se relaciona e vai além da sua individualidade e finitude" (DINIZ; KOLLER, 2010, p. 66).

Tendo em vista que as Representações Sociais influenciam diretamente na maneira como as pessoas se relacionam, trocam informações, conhecimentos e todos estes fatores interagem também com uma dimensão afetiva que existe e atravessa as Representações, com grande importância no funcionamento destas, sendo que os principais trabalhos de elaboração e consolidação da teoria reconhecem o papel da esfera emocional no funcionamento das representações, ou seja, significa dizer que, se consideramos que uma Representação é um conhecimento estruturado que tem um papel determinante no modo como os indivíduos veem e reagem face à realidade, fica evidente que este conhecimento é dotado de cargas afetivas e é ativado por um componente afetivo (CAMPOS; ROUQUETTE, 2003).

0 afeto traduz-se pela capacidade de 0 ser humano estabelecer relações e criar vínculos (DINIZ; KOLLER, 2010) e, segundo Coelho de Souza (2011), a construção do conhecimento pelos indivíduos está relacionada a operações cognitivas construídas a partir das emoções, estruturando o seu acervo cognitivo no início de suas vidas. Observa-se então a importância da família nos diferentes campos de desenvolvimento das pessoas desde seu nascimento até a fase adulta e, conforme aponta Pratta e Santos (2007), entre as funções psicológicas da família encontram-se a de proporcionar afeto e um ambiente adequado para a aprendizagem empírica que sustenta o processo de desenvolvimento cognitivo dos seres humanos, o que é fundamental para garantir a sobrevivência dos indivíduos.

Assim, fica evidente que a família é o pilar principal para o desenvolvimento das pessoas e que, devido ao seu componente afetivo, as representações sociais também estão influenciadas pela vivência familiar de qualquer grupo social e suas concepções sobre educação científica e escolar passam por estes componentes, determinando o produto final das representações.

\section{PERCURSO METODOLÓGICO}

Para 0 desenvolvimento deste estudo adotou-se a análise prototípica (CABECINHAS, 2003; WACHELKE, 2009; TAKAHASHI, 2018), constituída de uma etapa de coleta de evocações de palavras 
utilizando um questionário de evocação livre, seguida de uma análise destas evocações a partir de critérios específicos que serão descritos posteriormente.

Os participantes da pesquisa foram 71 estudantes do Ensino Fundamental Anos Finais de uma escola pública do interior do Estado do Paraná bem como 35 responsáveis (denominamos responsáveis respeitando as diferentes formações familiares dos alunos). A escola atende alunos dos bairros próximos, todos estes bairros possuem residências com porte de classe média e classe média baixa. Todos os estudantes que participaram da pesquisa tiveram aprovação de algum responsável. Entretanto, mesmo consentindo que seu filho (a) (ou outro grau de parentesco) participasse da pesquisa apenas metade dos responsáveis quis ou pode participar.

Segundo Sá (1996) as Representações Sociais são constituídas de um sistema interno duplo, contendo um sistema de representações centrais e outro de representações periféricas, cujas características variam, sendo a região central marcada por uma memória coletiva, que reflete valores e as condições sócio-históricas do grupo, tendo uma base comum e consensual coletivamente compartilhada, sendo estável, coerente e resistente à mudança, o que mantêm a estabilidade da Representação. Conforme colocam Amaral e Alves (2013) é o núcleo central que garante o significado da representação. 0 sistema periférico, por sua vez, promove uma conexão entre a realidade concreta $\mathrm{e}$ o sistema central, atualizando-0 e contextualizando-0 de forma a manter um caráter móvel, flexível e individualizante das Representações Sociais (SÁ, 1996).

Sendo assim, conforme argumentam Wachelke e Wolter (2011), uma das técnicas mais utilizadas para a caracterização da estrutura das Representações Sociais é a técnica de Análise Prototípica, para tal análise utilizamos como instrumento coleta de dados o Questionário de Evocação Livre e, para melhor caracterizar os participantes utilizamos um Questionário Sócio Cultural. Os dois instrumentos de coleta de dados foram avaliados intersubjetivamente pelos membros do grupo de pesquisa ao qual os autores deste trabalho participam. A coleta de dados com os estudantes ocorreu na própria escola e os responsáveis receberam os questionários para responder em casa.

0 Questionário Sócio Cultural foi organizado com 10 perguntas, contendo questões importantes para se estabelecer o perfil sociocultural dos participantes, principalmente no que se refere ao acesso à tecnologia e aos conteúdos científicos disponíveis em diferentes meios de informação, bem como caracterizar 0 ambiente de convívio social dos participantes da pesquisa.

0 Questionário de Evocação Livre atende ao que foi proposto por Vèrges (WACHELKE; WOLTER, 2011; TAKAHASHI, 2018) e foi adotado para a coleta das Representações Sociais dos participantes. Este questionário composto de três questões que precisam ser respondidas na ordem foi apresentado nesta pesquisa partindo da seguinte expressão indutora: "Diga cinco palavras ou expressões que vêm a sua cabeça quando se pensa em 'educação científica fora da escola', ou seja, aprender ciência fora da escola". Em seguida, pediu-se que os sujeitos dessem uma importância para uma das cinco expressões evocadas, classificando-as da mais importante para a menos importante.

Por fim, foi pedido que se determinasse a incondicionalidade de cada uma das cinco evocações, ou seja, se elas possuíam alta relação de significado com o tema proposto. Para tanto, foi dada a seguinte instrução: "é possível pensar em 'ciência no dia-a-dia' sem pensar na 'palavra ou expressão evocada'?”. Uma resposta negativa implica em uma avaliação de que o termo evocado faz referência a um elemento essencial da representação social, por estar atrelado diretamente ao tema proposto, ou seja, o sujeito não consegue pensar a "ciência no dia-a-dia" sem a evocação realizada. Os participantes tiveram que responder a essa questão para cada uma das evocações que eles apresentaram. 
A análise dos dados iniciou com a organização das respostas dos sujeitos. A análise dos dados dos alunos foi feita separada da análise das respostas dos responsáveis. Assim, as palavras evocadas foram agrupadas seguindo-se 0 critério de lematização, ou seja, colocando palavras que compartilhavam a mesma classe e radical, ou seja, que estão no plural e singular ou masculinas e femininas em um mesmo grupo. Por exemplo, no grupo AULA se adiciona as palavras "aula" e "aulas". No caso das expressões evocadas, o agrupamento ocorreu de acordo com critérios semânticos buscando-se significados comuns entre as expressões. Por exemplo, em um grupo semântico como ELETRICIDADE pode ser agrupada a palavra "eletricidade" e a expressão "energia elétrica".

Para a análise das evocações após o processo de agrupamento, primeiramente se calcula a frequência (f) das palavras evocadas em cada grupo semântico ( $f$ = número de aparições de uma evocação), bem como a frequência média do total das evocações ( $\mu \mathrm{f})$. Além disto, também é calculada a ordem média de evocação (OME), que consiste em uma relação entre a valoração das evocações por sua frequência e a média das OME ( $\mu$ OME).

Para o cálculo da valoração, foi atribuído um valor para cada palavra de acordo com a ordem de sua evocação, sendo que, a primeira palavra evocada tem mais valor que a última, portanto, para cada sujeito a primeira palavra evocada tem 0 valor 5 , a segunda 4, a terceira 3 , a quarta evocação valendo 2 e a quinta valendo 1. Somando-se a frequência das aparições de uma mesma palavra em cada valor se obtêm um grau de valoração. Por exemplo, a palavra "animais" foi evocada uma vez em primeiro (valor 5 ) e duas vezes em segundo (valor $4+4=8$ ) por três sujeitos diferentes, obtendo uma valoração igual a 13 e uma frequência de 3 aparições. A partir da relação entre esta valoração e frequência, 13 divido por 3, se obtêm uma OME de 4.33 .

Os valores médios encontrados para as evocações servem de base para colocá-las no quadrante de Vèrges (QUADRO 1), em que são agrupadas de acordo com sua frequência e OME, tendo como nota de corte a frequência média $(\mu \mathrm{F})$ das evocações e a média das ordens médias de evocação ( $\mu \mathrm{OME})$.

Quadro 1 - Quadrante de Vèrges utilizado para organização e análise das evocações emitidas nos questionários de evocação livre.

\begin{tabular}{|c|c|c|c|c|c|}
\hline \multicolumn{2}{|c|}{ ZONA DE NÚCLEO CENTRAL } & \multicolumn{3}{c|}{$1^{\text {a PERIFERIA }}$} \\
\hline \multicolumn{2}{|c|}{$\mathrm{f} \geq \mu \mathrm{f} /$ OME $<\mu$ OME } & G.S & $\mathrm{f} \geq \mu \mathrm{f} /$ OME $\geq \mu$ OME & OME \\
\hline G.S & $\mathrm{f}$ & OME & \multicolumn{3}{c|}{ 2 $^{\text {a PERIFERIA }}$} \\
\hline \multicolumn{2}{|c|}{} & & \multicolumn{3}{c|}{$\mathrm{f}<\mu \mathrm{f} /$ OME $\geq \mu$ OME } \\
\hline ELEMENTOS CONTRASTANTES $-3^{\circ}$ QUADRANTE & G.S & $\mathrm{f}$ & OME \\
\hline \multicolumn{2}{|c|}{$\mathrm{f}<\mu \mathrm{f} /$ OME $<\mu$ OME } & OME & & \\
\hline G.S & $\mathrm{f}$ & & & \\
\hline
\end{tabular}

(Fonte: adaptado de Takahashi, 2018. G.S. = grupo semântico; $f$ = frequência de evocações; $\mu \mathrm{f}=$ média das frequências de evocações; OME = ordem média das evocações; $\mu \mathrm{OME}=$ média da ordem média de evocações.)

Portanto, palavras que possuem um valor de f maior que $\mu \mathrm{f}$ e um valor de OME inferior à $\mu \mathrm{OME}$ são colocadas no primeiro quadrante, que se constitui o núcleo central da Representação. Aquelas evocações que apresentarem uma $\mathrm{f}$ e OME maiores que as suas respectivas médias são colocadas 
no segundo quadrante, em que se encontram os primeiros elementos intermediários. No terceiro quadrante, dos elementos intermediários contrastantes, são colocadas aquelas evocações onde F e OME são menores que a $\mu$ f e $\mu O M E$ e, por último, no quadrante dos elementos periféricos são colocadas as evocações com f menor que $\mu \mathrm{f}$ e OME maior que $\mu \mathrm{OME}$.

Como análise complementar à análise prototípica, foi calculado também o valor simbólico das evocações (VS), agrupando-se aqueles termos que possuem como resposta, simultaneamente, a condição de "muito importante" e "não" para a incondicionalidade, ou seja, que apresentam um alto valor simbólico para a representação, uma vez que são termos importantes e que não podem ser pensados fora do tema proposto, ou seja, a Representação dos sujeitos tem forte ligação com a palavra evocada.

Assim, para se determinar a valoração de cada evocação, foi realizado um cálculo que relaciona a frequência com que cada palavra foi evocada tendo um alto valor simbólico $\left(f_{v s}\right)$ dividindo pela frequência total das evocações em determinado grupo semântico $\left(f_{G S}\right)$. Seguindo o exemplo anterior, das três vezes $(f=3)$ que a palavra "animal" apareceu, 2 possuíam alto valor simbólico $\left(f_{v s}=2\right)$, recebendo resposta negativa à incondicionalidade e condição de muita importância. Assim, divide-se 2 por 3 e obtêm-se um resultado de 0.67 (VS = 0.67).

Esta análise complementar é importante, pois subsidia a análise prototípica ao confrontar a centralidade daquelas palavras que têm uma alta frequência e valoração com o seu valor simbólico, visto que estas evocações são muito importantes e incondicionais para a Representação.

\section{ANÁLISE DOS RESULTADOS}

A partir dos dados coletados pelo questionário sociocultural foi possível realizar este estudo com um total de 71 estudantes, com idade média de 14 anos, sendo 0 mais velho com 17 anos e 0 mais novo com 12. Destes estudantes, 40 eram do sexo masculino e 31 do sexo feminino, 25 se encontravam no sétimo ano e 46 no nono ano 0 que torna a amostra pertinente para a caracterização das representações deste grupo de sujeitos, uma vez que abrange estudantes da primeira metade do ciclo $\left(7^{\circ}\right.$ ano) e estudantes do final do ciclo $\left(9^{\circ}\right.$ ano) de estudos do Ensino Fundamental. Além disto, a amostra também engloba mais de $20 \%$ do total (303) de estudantes deste ciclo na escola em questão, o que torna o estudo significativo estatisticamente (CASTR0, 2004).

Dos 71 estudantes participantes, 31 (43\% da amostra) dividem suas residências com a mãe, pai e irmãos, enquanto, 9 (12\%) moram com a mãe e pai e sem a presença de irmãos e 9 moram somente com a mãe. Além disto, 5 (7\%) moram com a mãe e avós e 5 moram com a mãe e 0 padrasto, sendo que 2 (3\%) moram somente com o pai e outros 2 moram somente com os avós. Por fim, 1 estudante divide sua residência com o pai e a madrasta e 6 (8\%) estudantes, classificados em "outros", possuem outras variações na maneira como compartilham seus lares, havendo um caso de sujeitos que dividem a moradia com a mãe, avós, dois primos e um tio ou outro estudante que mora com o pai, madrasta, irmão e primo e, por esta razão, foram agrupados nesta categoria.

Ao se observar estes diferentes tipos de arranjos familiares, é importante destacar que os laços familiares se tornam mais vitais do que nunca para o sucesso das famílias em tempos atuais e a instabilidade nestas relações, como divórcios, brigas ou até mesmo o falecimento de algum dos cônjuges, tende a aumentar os riscos de adaptação e desajustamento dos filhos jovens frente as suas demandas sociais, como a convivência e o desempenho escolar (FOMBY; CHERLIN, 2007; WALSH, 2016). Contudo, em meio a estas turbulências, novos padrões de relacionamento são forjados dentro 
destas famílias com 0 intuito de se estabelecer bons laços de afeto entre os indivíduos, sendo que, estes arranjos familiares diferentes dos tradicionais "pai, mãe e filhos" podem também ter bons resultados no funcionamento e adequação de uma determinada família frente aos desafios impostos pela sociedade (WALSH, 2016).

Em relação ao acesso á tecnologia, constatou-se que os estudantes possuem amplo acesso aos principais meios de comunicação com uma média de 2 aparelhos televisores e 1 computador por residência. Além disto, 66 (93\%) estudantes disseram ter acesso também às principais formas de conexão com a rede internet em suas residências, sendo a conexão banda-larga/Wi-Fi a mais comum, mas também se nota uma presença relevante das conexões por redes móveis utilizadas em aparelhos celulares e tablets, sendo a conexão 4G mais frequente com 29 (41\%) estudantes possuindo acesso, seguida da conexão $3 \mathrm{G}$ com 3 (4\%) acessos. Cabe notar que o número de estudantes extrapolou o número da amostra pois, muitos dos participantes disseram possuir acesso a mais de uma forma de conexão, sendo que, nenhum afirmou não possuir nenhum tipo de acesso.

Um dado interessante é que apesar de possuírem amplo acesso aos meios de comunicação atuais, em resposta a questão do questionário sociocultural que questionava se havia o interesse por temas especificamente científicos, nenhum estudante afirmou acompanhar ou pesquisar temas com este tipo de conteúdo. Neste caso, apenas um sujeito manifestou interesse por uma série televisiva que aborda temas da Medicina.

Para os responsáveis não foram traçados os perfis de acesso a tecnologia e moradia visto que, em sua maioria, correspondem às mesmas informações para os estudantes, exceto que os adultos possuem maior acesso à internet eaos equipamentos eletrônicos. Contudo, é interessante analisar 0 perfil de idades e escolaridade destes sujeitos para se estabelecer o grau de aprofundamento científico em que se encontram, partindo do pressuposto que, quanto maior o grau de escolaridade destes indivíduos, maior o seu contato com os padrões de produção de conhecimento científico, que é mais desenvolvido no Ensino Superior que no Ensino Básico no Brasil.

Sendo assim, participaram da pesquisa 35 responsáveis, com idade média de 41 anos, sendo o mais novo com 26 e 0 mais velho com 74. Em relação à formação escolar, 26 responsáveis (75\%) possuem 0 ensino médio completo, sendo que destes, 10 possuem o Ensino Superior completo, com 2 responsáveis possuindo também uma pós-graduação completa. Por outro lado, temos 7 (20\%) responsáveis que ainda não terminaram 0 Ensino Médio e outros 2 (5\%) que não completaram o Ensino Fundamental.

Desta maneira, quanto à formação escolar, observa-se uma amostra heterogênea, com a maioria dos responsáveis possuindo pelo menos o Ensino Médio completo e alguns poucos que ainda não completaram o Médio e também o Fundamental. Assim os responsáveis, por serem as pessoas mais próximas, exercem grande influência nas Representações destes estudantes.

0 acesso e a interação com os meios de comunicação em massa estão presentes em grande parte da vida dos sujeitos aqui investigados, que utilizam a Televisão e a Internet para a obtenção da maioria de suas informações. Contudo, o foco deste trabalho não foi quantificar a influência dos veículos de informação, mas utilizar os dados colhidos para verificar uma possível relação com a construção das Representações aqui investigadas, pois concordamos com Morigi (2014) que a mídia e os veículos de comunicação em massa são componentes culturais que exercem grande importância na construção de Representações Sociais.

Portanto, as representações sobre Ciência Informal dos estudantes e responsáveis podem ter recebido influência das informações propagadas pela mídia, principalmente a internet, meio de comunicação amplamente utilizado pelos indivíduos aqui investigados, pois, conforme aponta 
Flores (2012, p. 1), "independente do canal no qual são veiculadas, as notícias e reportagens sobre Ciência produzem representações e valores sobre a atividade científica."

Partindo para a análise prototípica, com os estudantes foi possível capturar 354 evocações, com 70 indivíduos evocando 5 palavras/expressões e 1evocando somente 4. Após a exclusão das evocações com frequência insignificante igual a 1, ou seja, aquelas que foram evocadas somente uma vez (TAKAHASHI, 2018), restaram 224 evocações. Destas evocações, muitas apresentaram frequência igual a 2, ou seja, menor que 1\% (2.24) da amostra significativa total e foram descartadas, por serem consideradas também com baixa relevância estatística, evitando assim termos que possam descaracterizar o núcleo central devido a recorrência de variadas formas com baixa frequência, o que altera as médias de corte tanto para as frequências, quanto para as ordens médias de evocação (WACHELKE E WOLTER, 2011).

Sendo assim, restaram 156 evocações contidas em 29 Grupos Semânticos, organizados de acordo com os critérios semânticos discutidos no percurso metodológico e as notas de referência utilizadas para a construção do quadrante foram a frequência média, com valor $5.38(\mu \mathrm{f}=5.38)$ e a média das OME, com valor $3.16(\mu \mathrm{OME}=3.16)($ TABELA 1), sendo importante observar que quanto maior for o valor da "f" e menor o valor da "OME", mais importância e contribuiç̧ão possui a evocação para a representação social estudada (PAULA, 2012).

Portanto, a partir destes dados, as evocações que possuem $\mathrm{f} \geq 5.38$ e OME $<3.16$ e podem ser adicionadas no primeiro quadrante dos elementos centrais, local em que se encontram os elementos de um possível núcleo central das Representações destes indivíduos, são os grupos "vida", "tecnologia", "pesquisas", "energia" e "estudar" (QUADRO 2).

Nos elementos intermediários próximos do núcleo, ou seja, aqueles elementos com $\mathrm{f} \geq 5.38$ e OME $\geq 3.16$, ficaram os grupos "animais", "plantas", "natureza", "experiências", "luz", "água" e "escola". No quadrante dos elementos intermediários distantes do núcleo, ou seja, com f $<5.38$ e OME < 3.16 ficaram os grupos "ar", "carro", "substâncias", "sol", "células", "doenças", "alimentar" e "misturas". Por fim, nos elementos periféricos, nos quais se enquadram as evocações com $f<5.38$ e OME $\geq 3.16$ foram adicionados os grupos "celular", "bactérias", "corpo humano", "aulas", "química", "eletricidade", "preguiça", "colégio" e "insetos" (QUADRO 2).

Desta forma, ao observar o todo da estrutura destas evocações no quadrante, se evidencia uma imagem sobre Ciência Informal, mais próxima do que é a Ciência Formal, ao evocarem por todas as regiões termos como tecnologias e pesquisas na região central e experiências, carro e celular, no primeiro, segundo e terceiro quadrante, respectivamente.

A segunda imagem de Ciência Informal que estes indivíduos demonstraram também está vinculada à Ciência Formal, podendo ser observada em todas as outras evocações, que estão relacionadas ao ambiente formal da Escola, podendo ser dividido em duas esferas, uma contendo os conteúdos escolares condizentes com a faixa etária destes estudantes, como é exemplificado no primeiro quadrante com as evocações vida e energia, conteúdos de sétimos e nonos anos do Ensino Fundamental, respectivamente. Nos quadrantes periféricos termos semelhantes também foram abundantes, como é o caso de luz, ar, substâncias, misturas, animais e plantas. Além disto, também podem ser observadas evocações vinculadas aos procedimentos relacionados ao cotidiano escolar, como demonstram os elementos estudar no quadrante central, escola, colégio, aulas e preguiça nos elementos periféricos. 
Tabela 1 - Ordem (Valor), Frequência (f), Valoração (V) e Ordem Média de Evocação (OME) das evocações realizadas por estudantes de uma escola na cidade de Londrina, Paraná, Brasil, a partir do termo indutor "ciência no dia-a-dia".

\begin{tabular}{|c|c|c|c|c|c|c|c|c|}
\hline \multirow{2}{*}{$\begin{array}{c}\text { EVOCAÇÕES } \\
\text { (G. S.) }\end{array}$} & \multicolumn{5}{|c|}{ ORDEM DE EVOCAÇÃ̃O (VALOR) } & \multirow{2}{*}{ v } & \multirow{2}{*}{$\frac{f}{(>1 \mathrm{e}>1 \%)}$} & \multirow{2}{*}{ OME } \\
\hline & 1 (5) & $2(4)$ & $3(3)$ & $4(2)$ & $5(1)$ & & & \\
\hline ANIMAIS & 5 & 1 & 3 & 5 & 1 & 49 & 15 & 3.27 \\
\hline PLANTAS & 4 & 1 & 3 & 2 & 1 & 38 & 11 & 3.45 \\
\hline NATUREZA & 4 & 2 & 2 & 1 & 1 & 37 & 10 & 3.70 \\
\hline EXPERIÊNCIAS & 3 & 3 & 3 & 0 & 1 & 37 & 10 & 3.70 \\
\hline VIDA & 2 & 1 & 0 & 2 & 2 & 20 & 7 & 2.86 \\
\hline TECNOLOGIA & 1 & 2 & 0 & 1 & 2 & 17 & 6 & 2.83 \\
\hline PESQUISAS & 0 & 1 & 1 & 2 & 2 & 13 & 6 & 2.16 \\
\hline LUZ & 2 & 1 & 1 & 1 & 1 & 20 & 6 & 3.33 \\
\hline ENERGIA & 1 & 0 & 1 & 0 & 4 & 12 & 6 & 2.00 \\
\hline ÁGUA & 3 & 1 & 0 & 0 & 2 & 21 & 6 & 3.50 \\
\hline ESTUDAR & 1 & 1 & 1 & 3 & 0 & 18 & 6 & 3.00 \\
\hline ESCOLA & 2 & 1 & 1 & 1 & 1 & 20 & 6 & 3.33 \\
\hline$A R$ & 0 & 1 & 2 & 2 & 0 & 12 & 5 & 2.40 \\
\hline CELULAR & 2 & 1 & 0 & 1 & 1 & 17 & 5 & 3.40 \\
\hline BACTÉRIAS & 2 & 3 & 0 & 0 & 0 & 22 & 5 & 4.40 \\
\hline CORPO HUMANO & 2 & 2 & 1 & 0 & 0 & 21 & 5 & 4.20 \\
\hline AULAS & 1 & 2 & 0 & 0 & 1 & 14 & 4 & 3.50 \\
\hline CARRO & 0 & 0 & 2 & 1 & 1 & 9 & 4 & 2.25 \\
\hline SUBSTÂNCIAS & 0 & 0 & 0 & 0 & 3 & 3 & 3 & 1.00 \\
\hline SOL & 0 & 1 & 1 & 0 & 1 & 8 & 3 & 2.67 \\
\hline CÉLULAS & 1 & 0 & 0 & 1 & 1 & 8 & 3 & 2.67 \\
\hline QUÍMICA & 1 & 1 & 0 & 1 & 0 & 11 & 3 & 3.67 \\
\hline ELETRICIDADE & 1 & 2 & 0 & 0 & 0 & 13 & 3 & 4.33 \\
\hline PREGUIÇA & 2 & 1 & 0 & 0 & 0 & 14 & 3 & 4.67 \\
\hline DOENÇAS & 0 & 1 & 1 & 0 & 2 & 9 & 3 & 3.00 \\
\hline ALIMENTAR & 0 & 1 & 1 & 0 & 1 & 8 & 3 & 2.67 \\
\hline COLÉGIO & 1 & 1 & 0 & 0 & 1 & 10 & 3 & 3.33 \\
\hline MISTURAS & 0 & 0 & 2 & 1 & 0 & 8 & 3 & 2.67 \\
\hline INSETOS & 1 & 1 & 0 & 1 & 1 & 12 & 3 & 4.00 \\
\hline TOTAL & & & & & & & 156 & \\
\hline MÉDIA & & & & & & & 5.38 & 3.17 \\
\hline
\end{tabular}

(Fonte: elaborado pelos autores após aplicação de questionário de evocação livre) 
Quadro 2 - Estrutura das Representações Sociais dos estudantes a partir do termo indutor "educação científica fora da escola".

\begin{tabular}{|c|c|c|c|c|c|}
\hline \multicolumn{3}{|c|}{ Elementos Centrais $-1^{\circ} \mathrm{Q}$. } & \multicolumn{3}{|c|}{ Elementos Intermediários $-2^{\circ} Q$} \\
\hline \multicolumn{3}{|c|}{$\mathrm{f} \geq 5.38-\mathrm{OME}<3.16$} & \multicolumn{3}{|c|}{$\mathrm{f} \geq 5.38-\mathrm{OME} \geq 3.16$} \\
\hline G.S. & $f$ & OME & G. S. & $\mathrm{f}$ & OME \\
\hline & & & ANIMAIS & 15 & 3.27 \\
\hline VIDA & 7 & 2.86 & PLANTAS & 11 & 3.45 \\
\hline TECNOLOGIA & 6 & 2.83 & NATUREZA & 10 & 3.70 \\
\hline PESQUISAS & 6 & 2.16 & EXPERIÊNCIAS & 10 & 3.70 \\
\hline ENERGIA & 6 & 2.00 & LUZ & 6 & 3.33 \\
\hline \multirow[t]{2}{*}{ ESTUDAR } & 6 & 3.00 & ÁGUA & 6 & 3.50 \\
\hline & & & ESCOLA & 6 & 3.33 \\
\hline \multicolumn{3}{|c|}{ ElementosIntermediários - $3^{\circ} \mathrm{Q}$. } & \multicolumn{3}{|c|}{ ElementosPeriféricos - $4^{\circ} \mathrm{Q}$. } \\
\hline \multicolumn{3}{|c|}{$\mathrm{f}<5.38-\mathrm{OME}<3.16$} & \multicolumn{3}{|c|}{$\mathrm{f}<5.38-\mathrm{OME} \geq 3.16$} \\
\hline G.S. & $f$ & OME & G.S. & $f$ & OME \\
\hline AR & 5 & 2.40 & CELULAR & 5 & 3.40 \\
\hline CARRO & 4 & 2.25 & BACTÉRIAS & 5 & 4.40 \\
\hline SUBSTÂNCIAS & 3 & 1.00 & CORPO HUMANO & 5 & 4.20 \\
\hline SOL & 3 & 2.67 & AULAS & 4 & 3.50 \\
\hline CÉLULAS & 3 & 2.67 & QUÍMICA & 3 & 3.67 \\
\hline DOENÇAS & 3 & 3.00 & ELETRICIDADE & 3 & 4.33 \\
\hline ALIMENTAR & 3 & 2.67 & PREGUIÇA & 3 & 4.67 \\
\hline MISTURAS & 3 & 2.67 & COLÉGIO & 3 & 3.33 \\
\hline & & & INSETOS & 3 & 4.00 \\
\hline
\end{tabular}

(Fonte: elaborado pelos autores após realização da análise prototípica)

Este resultado se assemelha ao encontrado por Petter (2011) que, ao buscar as Representações de estudantes das séries iniciais do Ensino Fundamental sobre o termo "Ciência", observou que eles a representam como "estudo", com uma noção de corpo humano, de "estudo" de doenças para alívio e bem estar das pessoas. Em contraste com a visão voltada aos conteúdos e à escola, há também na região central da Representação uma ideia de tecnologia, com elementos distantes incorporando itens tecnológicos como o carro e o aparelho celular. Resultado muito semelhante foi observado por Bortolai et al., (2016) que encontrou Representações de "Ciência" em estudantes de Ensino Médio, relacionadas ao corpo humano e elementos da natureza como animais, plantas, vida e célula, além de elementos como tecnologia e professor, mostrando o papel e correlação que os estudantes fazem dos elementos escola e tecnologia como importantes para seu desenvolvimento.

No mesmo sentido, com a análise complementar a partir daquelas evocações com alto valor simbólico para o termo "ciência no dia-a-dia", se observa que alguns dos grupos do núcleo central e dos elementos intermediários próximos também foram considerados muito importantes e incondicionais (Tabela 2). Por exemplo, considerando que os elementos mais importantes e, portanto, mais centrais nesta análise, são aqueles que possuem grau de valoração superior à 0.51 , ou seja, maior que 0 valor intermediário entre 1 e 0, e comparando com os termos observados na análise prototípica, observa-se a recorrência dos grupos "vida", "tecnologia", "energia" e "estudar" presentes na região 
do núcleo central do quadrante, ou seja, as expressões com alta valoração e frequência. No mesmo sentido, temos ainda os grupos "natureza", "luz" e "experiências" pertencentes ao quadrante da alta frequência e baixa valoração, ou seja, o segundo quadrante dos elementos intermediários próximos.

Tabela 2 - Valor Simbólico (VS) das evocações de estudantes dos anos finais do Ensino Fundamental a partir da taxa entre as frequências das palavras com alto valor simbólico $\left(f_{v s}\right)$ e a frequência total das palavras significativas $\left(f_{G S}\right)$ e mencionadas por pelo menos $5 \%$ dos participantes $(F \geq 4)$.

\begin{tabular}{|c|c|c|c|c|}
\hline EVOCAÇÃO & $f_{v s}$ & $f_{G S}$ & vS & Localização no Quadrante \\
\hline NATUREZA & 8 & 10 & 0.80 & $2^{\circ} Q$ \\
\hline AR & 4 & 5 & 0.80 & $3^{\circ} Q$ \\
\hline BACTÉRIAS & 4 & 5 & 0.80 & $4^{0} Q$ \\
\hline CORPO HUMANO & 4 & 5 & 0.80 & $4^{\circ} Q$ \\
\hline VIDA & 5 & 7 & 0.71 & $1^{\circ} \mathrm{Q}$. \\
\hline LUZ & 4 & 6 & 0.67 & $2^{\circ} Q$ \\
\hline ENERGIA & 4 & 6 & 0.67 & $1^{0} Q$ \\
\hline TECNOLOGIA & 4 & 6 & 0.67 & $1^{0} Q$ \\
\hline ESTUDAR & 4 & 6 & 0.67 & $1^{0} Q$ \\
\hline EXPERIÊNCIAS & 6 & 10 & 0.60 & $2^{\circ} Q$ \\
\hline ÁGUA & 3 & 6 & 0.50 & $2^{\circ} Q$ \\
\hline PESQUISAS & 3 & 6 & 0.50 & $1^{0} Q$. \\
\hline AULAS & 2 & 4 & 0.50 & $4^{0} Q$ \\
\hline CELULAR & 2 & 5 & 0.40 & $4^{\circ} Q$ \\
\hline PLANTAS & 4 & 11 & 0.36 & $2^{\circ} Q$ \\
\hline ANIMAIS & 5 & 15 & 0.33 & $1^{0} Q$ \\
\hline CARRO & 1 & 4 & 0.25 & $3^{\circ} Q$ \\
\hline ESCOLA & 1 & 6 & 0.17 & $2^{\circ} \mathrm{Q}$. \\
\hline
\end{tabular}

(Fonte: elaborado pelos autores após análise dos questionários de evocação livre)

Reforçando o caminho destas Representações para o conteúdo escolar, surgem termos muito importantes, vinculados aos conteúdos e que apareceram nas zonas mais distantes do quadrante, como por exemplo, os grupos "bactérias" e "corpo humano" que apresentaram elevados graus de importância, chegando a 0.81, mas se encontram no quadrante dos elementos periféricos distantes do núcleo central.

Assim, é interessante analisar também as Representações dos responsáveis para encontrar possíveis relações entre suas formas de simbolizar a Ciência Informal e a forma como seus filhos simbolizam, observando a influência do elemento grupal que é a família para a Representação.

Portanto, no caso dos responsáveis, foram realizadas 173 evocações, sendo que dos 35 participantes, um fez apenas 3 evocações. Destas evocações, foram descartadas aquelas com frequência menor que 1 e menor que $2 \%$ (2.16) da amostra total dos responsáveis. Aqui o valor foi modificado de $1 \%$ para $2 \%$ porque a amostra dos responsáveis foi menor que a dos estudantes, e isto evita a recorrência de evocações com baixa relevância estatística de acordo com a amostra. Desta maneira, restaram para análise 76 evocações organizadas em 17 grupos semânticos diferentes (TABELA 3). 
Tabela 3 - Ordem (Valor), Frequência (f), Valoração (V) e Ordem Média de Evocação (OME) das evocações realizadas por responsáveis por estudantes de uma escola na cidade de Londrina, Paraná, Brasil, a partir do termo indutor "ciência no dia-a-dia".

\begin{tabular}{|c|c|c|c|c|c|c|c|c|}
\hline \multirow{2}{*}{ EVOCAÇÕES } & \multicolumn{5}{|c|}{ IMPORTÂNCIA (VALOR) } & \multirow{2}{*}{ V } & \multirow{2}{*}{$\frac{f}{(>1 \text { e }>2 \%)}$} & \multirow{2}{*}{ OME } \\
\hline & $1(5)$ & $2(4)$ & $3(3)$ & $4(2)$ & $5(1)$ & & & \\
\hline CONHECIMENTO & 3 & 2 & 2 & 1 & 3 & 34 & 11 & 3.09 \\
\hline EXPERIÊNCIAS & 1 & 1 & 2 & 2 & 2 & 21 & 8 & 2.62 \\
\hline PESQUISA & 2 & 2 & 0 & 2 & 1 & 23 & 7 & 3.28 \\
\hline IMPORTANTE & 3 & 2 & 0 & 0 & 1 & 24 & 6 & 4.00 \\
\hline APRENDIZAGEM & 0 & 1 & 2 & 1 & 1 & 13 & 5 & 2.60 \\
\hline DESCOBERTA & 1 & 1 & 0 & 1 & 1 & 12 & 4 & 3.00 \\
\hline ASSISTIR TV & 0 & 2 & 1 & 1 & 0 & 13 & 4 & 3.25 \\
\hline TECNOLOGIA & 0 & 3 & 1 & 0 & 0 & 15 & 4 & 3.75 \\
\hline FAMÍLIA & 0 & 0 & 0 & 2 & 1 & 5 & 3 & 1.67 \\
\hline CORPO HUMANO & 0 & 0 & 1 & 1 & 1 & 6 & 3 & 2.00 \\
\hline LIVROS & 1 & 1 & 0 & 1 & 0 & 11 & 3 & 3.67 \\
\hline CULTURA & 1 & 0 & 1 & 0 & 1 & 9 & 3 & 3.00 \\
\hline CULINÁRIA & 2 & 0 & 0 & 0 & 1 & 11 & 3 & 3.67 \\
\hline INFORMAÇÃO & 0 & 0 & 1 & 2 & 0 & 7 & 3 & 2.33 \\
\hline OBSERVAÇÃO & 3 & 0 & 0 & 0 & 0 & 15 & 3 & 5.00 \\
\hline INTERNET & 1 & 0 & 2 & 0 & 0 & 11 & 3 & 3.67 \\
\hline NATUREZA & 1 & 0 & 1 & 1 & 0 & 10 & 3 & 3.33 \\
\hline TOTAL & & & & & & & 76 & \\
\hline MÉDIA & & & & & & & 4.47 & 3.17 \\
\hline
\end{tabular}

(Fonte: elaborado pelos autores após aplicação de questionário de evocação livre)

0 grupo semântico que apresentou maior frequência foi o grupo "conhecimento", com frequência 11, seguido por "experiências" com frequência 8. Em seguida está 0 grupo semântico "pesquisa" com frequência 7, seguido pelo grupo semântico "importante" com frequência 6 . Foram evocados ainda palavras no grupo semântico "aprendizagem" com uma frequência 5, os grupos "descoberta", "assistir TV" e "tecnologia", todos com frequência 4 e, por fim, os grupos "família", "corpo humano", "livros", "cultura", "culinária", "informação", "observação", "internet" e "natureza", todos com frequência 3.

No caso dos responsáveis as notas de referência para a construção do quadrante foram a frequência média de 4.47 e a média das ordens médias de evocação com valor 3.17. As evocações de alta frequência e valoração constituindo do núcleo central foram os grupos "conhecimento", "experiências" e "aprendizagem". No quadrante dos elementos intermediários próximos do núcleo central, se enquadram as evocações de alta frequência e baixa valoração, no caso os grupos "pesquisa" e "importante". No grupo das evocações de baixa frequência e alta valoração, ou seja, os elementos intermediários distantes do núcleo central encontram-se os grupos "descoberta", "família", "corpo humano", "cultura" e "informação". Por fim, no quadrante dos elementos periféricos da representação onde se encaixam elementos de baixa frequência e valoração, encontram-se os grupos "assistir TV”, "tecnologia”, "livros", "culinária”, "observação", "internet” e "natureza” (QUADRO 3). 
Quadro 3 - Estrutura das Representações Sociais dos responsáveis a partir do termo indutor "educação científica fora da escola".

\begin{tabular}{|c|c|c|c|c|c|}
\hline \multicolumn{3}{|c|}{ Elementos Centrais $-1^{\circ} Q$. } & \multicolumn{3}{|c|}{ Elementos Intermediários $-2^{\circ} \mathrm{Q}$} \\
\hline \multicolumn{3}{|c|}{$\mathrm{f} \geq 4.47-\mathrm{OME}<3.17$} & \multicolumn{3}{|c|}{$\mathrm{f} \geq 4.47-\mathrm{OME} \geq 3.17$} \\
\hline G. S. & $f$ & OME & G. S. & $f$ & OME \\
\hline CONHECIMENTO & 11 & 3.09 & PESQUISA & 7 & 3.28 \\
\hline EXPERIÊNCIAS & 8 & 2.62 & IMPORTANTE & 6 & 4.00 \\
\hline APRENDIZAGEM & 5 & 2.60 & & & \\
\hline \multicolumn{3}{|c|}{ Elementos Intermediários - $3^{\circ} \mathrm{Q}$. } & \multicolumn{3}{|c|}{ Elementos Periféricos $-4^{\circ} \mathrm{Q}$. } \\
\hline \multicolumn{3}{|c|}{$\mathrm{f}<4.47-0 \mathrm{ME}<3.17$} & \multicolumn{3}{|c|}{$\mathrm{f}<4.47-\mathrm{OME} \geq 3.17$} \\
\hline G. S. & $f$ & OME & G.S. & $f$ & OME \\
\hline DESCOBERTA & 4 & 3.00 & ASSISTIR TV & 4 & 3.25 \\
\hline FAMÍLIA & 3 & 1.67 & TECNOLOGIA & 4 & 3.75 \\
\hline CORPO HUMANO & 3 & 2.00 & LIVROS & 3 & 3.67 \\
\hline CULTURA & 3 & 3.00 & CULINÁRIA & 3 & 3.67 \\
\hline \multirow[t]{3}{*}{ INFORMAÇÃO } & 3 & 2.33 & OBSERVAÇÃO & 3 & 5.00 \\
\hline & & & INTERNET & 3 & 3.67 \\
\hline & & & NATUREZA & 3 & 3.33 \\
\hline
\end{tabular}

(Fonte: elaborado pelos autores após a realização da análise prototípica)

Desta maneira, se observa que as Representações de Ciência Informal dos responsáveis possuem aspectos diferentes das dos estudantes, indo em direção a uma concepção de ciência no dia-a-dia voltada para o conhecimento, troca de experiências, vinculados com a pesquisa e aprendizagem. Além disto, os responsáveis consideram a ciência no dia-a-dia como importante para 0 conhecimento e troca de experiências. Nos elementos mais distantes da Representação notam-se aspectos importantes que podem auxiliar na manutenção dos elementos mais fixos do núcleo central, como o caso da descoberta e cultura que podem ser considerados próximos do conhecimento, assim como a cultura e a família podem se aproximar da pesquisa e aprendizagem. Além disto, elementos como livros, tecnologia, assistir TV, também podem ser vistos como um ambiente de acesso ao conhecimento.

$\mathrm{Na}$ análise de importância das Representações, observa-se a recorrência dos termos que se encaixaram no núcleo central, com os grupos "conhecimento", "experiências" e "aprendizagem" apresentando também um grande valor simbólico, aproximando-se de 1, evidenciando que, além de possuírem uma alta frequência e valoração, estes grupos também possuem elevado valor simbólico, sendo importantes incondicionais para o tema ciência no dia-a-dia (TABELA 4). 
Tabela 4 - Valor simbólico (VS) das evocações dos responsáveis por estudantes dos anos finais do Ensino Fundamental a partir da taxa entre as frequências das palavras com alto valor simbólico $\left(f_{v s}\right)$ e a frequência total das palavras significativas $\left(f_{G S}\right)$ e mencionadas por pelo menos $5 \%$ dos participantes $(F \geq 3.8)$.

\begin{tabular}{ccccc}
\hline EVOCAÇÃO & $\mathrm{f}_{\mathrm{vS}}$ & $\mathrm{f}_{\mathrm{GS}}$ & VS & Localização no quadrante \\
CONHECIMENTO & 10 & 11 & 0.90 & $1^{\circ} \mathrm{Q}$. \\
EXPERIÊNCIAS & 7 & 8 & 0.87 & $1^{\circ} \mathrm{Q}$. \\
IMPORTANTE & 5 & 6 & 0.83 & $2^{\circ} \mathrm{Q}$. \\
APRENDIZAGEM & 4 & 5 & 0.80 & $1^{\circ} \mathrm{Q}$. \\
DESCOBERTA & 3 & 4 & 0.75 & $3^{\circ} \mathrm{Q}$. \\
PESQUISA & 5 & 7 & 0.71 & $2^{\circ} \mathrm{Q}$. \\
ASSISTIR TV & 2 & 4 & 0.50 & $4^{0} \mathrm{Q}$. \\
TECNOLOGIA & 2 & 4 & 0.50 & $4^{\circ} \mathrm{Q}$. \\
\hline
\end{tabular}

(Fonte: elaborado pelos autores após análise dos questionários de evocação livre)

Por outro lado, alguns dos elementos mais distantes e com significados semânticos próximos aos elementos centrais também aparecem com elevado valor simbólico, como é o caso dos grupos "descoberta" e "pesquisa" que podem complementar a ideia de conhecimento, experiências e aprendizagem enquadrados no núcleo central desta Representação.

Por fim, os grupos "assistir TV" e "tecnologia" também apareceram com importância mediana, ambos com valor 0.50, indicando que metade das evocações enquadradas nestes grupos recebeu grande importância e foram incondicionais para o tema proposto, mostrando relação também com a região central, uma vez que estes sujeitos veem a TV e a Tecnologia como instrumentos importantes para a obtenção e troca de conhecimentos.

Em contraste com os estudantes, as Representações dos responsáveis parecem ter recebido influências de seu ambiente de convívio e aqui o papel da mídia nesta construção parece ser mais evidente, visto que, estamos na era da comunicação, com ferramentas de pesquisa e obtenção de conhecimentos eficientes, como o caso do Google, e sites semelhantes. Além disto, Representações vinculadas com uma noção de família e culinária parecem estar mais relacionadas à faixa etária e função social que os responsáveis possuem, sendo que, os jovens não estão demasiadamente preocupados com a organização de suas atividades ou ainda não enxergam o ambiente familiar como elemento importante para o seu aprendizado científico.

Conforme argumenta Petter (2011), por serem socialmente construídas, as Representações auxiliam na constituição de uma realidade comum, permitindo a comunicação entre os indivíduos. Assim, aspectos como estes auxiliam a demonstrar como os meios de comunicação e 0 ambiente de convívio de cada indivíduo podem modelar suas experiências e suas percepções de mundo, sendo que, as Representações também podem se modificar ao longo do tempo e de acordo com a vivência de cada indivíduo.

Assim como o processo de comunicação é importante na construção das Representações Sociais dos indivíduos, ele ainda se faz na construção da identidade dos sujeitos, estando as Representações na origem e ao longo de todo o processo de formação da identidade dos sujeitos (VALA, 1997) e assim, ao observarmos as Representações e o contexto de acesso à comunicação e as respostas aos questionários de evocação livre, pode-se dizer que 0 ambiente de convivência dos 
indivíduos tem forte influência na construção de suas Representações, visto que, as Representações dos estudantes estão ligadas ao ambiente escolar enquanto que, para os responsáveis, que em geral seguem uma rotina de trabalho e cuidado com os filhos, as Representações parecem estar mais ligadas com o acesso aos meios de comunicação, que é muito presente na vida destes indivíduos e a aspectos relacionados à vivência doméstica, como a culinária. Além disto, outro aspecto que merece ser observado é como as Representações podem influenciar o cotidiano dos indivíduos, visto que, os estudantes representam a Ciência Informal vinculada com a Escola, elemento que pode afastar estes sujeitos do comportamento científico e da compreensão dos elementos científicos cotidianos.

\section{CONSIDERAÇÕES FINAIS}

No início desta pesquisa foi indagado se a família e a escola podem influenciar na construção das Representações Sociais de Ciência no dia-a-dia dos estudantes. Assim, as respostas dos indivíduos para esta pesquisa demonstraram que 0 ambiente escolar é a principal forma de representação de Ciência Informal para os estudantes participantes, enquanto as representações dos responsáveis foram mais amplas, englobando elementos voltados ao conhecimento, aprendizagem e as trocas de experiências entre as pessoas.

Portanto, os estudantes possuem ainda uma visão da Ciência Informal, mais voltada para a Ciência Formal, como demonstram as evocações agrupadas na região do núcleo central. Por outro lado, os responsáveis possuem uma visão mais próxima do que é a Ciência Informal, englobando trocas de experiências e conhecimentos vinculados com as atividades diárias como assistir televisão e cozinhar, por exemplo.

Conforme coloca Sá (1996), as representações sociais têm um caráter estável, mas também mutável, podendo ser rígidas ou flexíveis e, sendo assim, as representações dos estudantes aqui investigados provavelmente sofrerão modificações e influências de outros contextos e do cotidiano futuro que passarão a conviver em ambientes diferentes da Escola.

Por outro lado, não se vê muitos elementos que demonstrem a família como influenciadora neste processo. Estes jovens consideram a Escola importante para 0 seu desenvolvimento e a relacionam com a Pesquisa e com a Ciência, sendo este ambiente um local importante na construção da identidade destes indivíduos.

Desta maneira, retomando o objetivo de verificar as Representações dos estudantes e de seus responsáveis sobre Ciência Informal, observa-se que 0 valor simbólico atribuído à Ciência Informal se relaciona com 0 ambiente escolar e uma imagem tecnicista da Ciência Formal. Portanto, cabe questionar por que estes indivíduos não possuem esse vínculo com o conhecimento informal e qual o papel da família nestas representações. Parece que as famílias se eximiram da responsabilidade de ensinar aspectos da ciência no dia-a-dia, deixando somente a escola como provedora destes conhecimentos.

Sendo assim, esta pesquisa apresenta um auxílio no entendimento da visão de Ciência Informal dos estudantes, a partir da realidade desta escola e pode servir como ajuda para estudos futuros na área de Educação em Ciência, que podem utilizar estas informações para compararem com estudantes de outras regiões e também para aproximar os conteúdos e os métodos de ensino dos professores em relação a seus estudantes. Além disto, a explanação inicial das representações dos responsáveis é de grande importância ao demonstrar a participação destes sujeitos na educação dos seus filhos, na construção de sua identidade e em sua visão de mundo. 


\section{REFERÊNCIAS}

ALVES-MAZZOTTI, A. J. Representações Sociais: aspectos teóricos e aplicações à Educação. Em Aberto, Brasília, n. 61, p. 60-74, 1994.

AMARAL, L. S; ALVES, M. S. Nó ou Núcleo central e esquema periférico. Cadernos CESPUC, n. 23, p. 30-36, 2013.

ARRUDA, A. Teoria das representações sociais e teorias de gênero. Cadernos de Pesquisa, [Online], n. 117, p. 127-147, 2002. Disponível em: <http://www.scielo.br/> Acesso em: 28 ago. 2019.

BORTOLAI, M. M. S et al. Núcleo central e periferia das Representações Sociais de alunos do Ensino Médio sobre Ciência. In: Encontro Nacional de Ensino de Química, n. 18, 2016, Florianópolis. Anais [...]. Universidade Federal de Santa Catarina: UFSC, 2016, p. 1-12.

BREAKWELL, G. M. Social Representation and Social Identity. Papers on Social Representations, Londres, n. 3, v. 2, p. 1-20, 1993.

CABECINHAS, R. Investigar representações sociais: metodologias e níveis de análise. In: BAPTISTA, M. M. Cultura: metodologias e investigação. 1 ed. Lisboa: Ver o Verso, 2009, p. 51-66.

CAMPOS, P. H. F; ROUQUETTE, M. L. Abordagem Estrutural e Componente Afetivo das Representações Sociais. Psicologia: Reflexão e Crítica, v. 16, n. 3, p. 435-445, 2003.

CARVALHO, M. E. P. Relações entre família e escola e suas implicações de gênero. Cadernos de Pesquisa, n. 110, p. $143-155,2000$.

CASCAIS, M. G. A; TERÁN, A. F. Educação formal, informal e não formal em Ciências: contribuições dos diversos espaços educativos. In: ENCONTRO DE PESQUISA EDUCACIONAL NORTE NORDESTE, n. 20, 2011, Manaus. Anais [...]. Universidade federal do Amazonas: UFAM, p. 1-9, 2011.

CASTRO, S. M. V. Representação social de Ciência de estudantes do ensino fundamental da Rede Municipal de Belém. 2004. 91 f. Dissertação (Mestrado em Educação em Ciências e Matemáticas) - Núcleo Pedagógico de apoio ao desenvolvimento científico da Universidade Federal do Pará, Universidade Federal do Pará, Belém, 2004.

COELHO DE SOUZA, M. T. C. As relações entre afetividade e inteligência no desenvolvimento psicológico. Psicologia: Teoria e Pesquisa, Brasília, v. 27, n. 2, p. 249-254, 2011.

DINIZ, E; KOLLER, S. H. 0 afeto como um processo de desenvolvimento ecológico. Educar, Curitiba, n. 36, p. 65-76, 2010.

FLORES, N. Divulgação de ciência na mídia: algumas reflexões. In: Intercom - Sociedade Brasileira de Estudos Interdisciplinares da Comunicação - Congresso de Ciências da Comunicação na Região Nordeste, n. 14, 2012, Recife. Anais [...]. Universidade Federal de Pernambuco: ed. 14, 2012, p. 1-12.

FOMBY, P; CHERLIN, A. J. Family instability and child well-being. American SociologicalReview, n. 72, p. 181-204, 2007. Disponível em: https://bit.ly/3eZteg8. Acesso em: 21 jul. 2020. 
GASPAR, A. A educação formal e a educação informal em ciências. In: MASSARANI, L. et al (Org.). Ciência e público: caminhos da divulgação científica no Brasil. 1 ed. Rio de Janeiro: Casa da Ciência - Centro Cultural de Ciência e Tecnologia/UFRJ, v. 1, p. 171-183, 2002.

MARQUES, M. 0. Educação nas ciências: interlocução e complementaridade. 1. ed. São Geraldo: Unijuí, 2002, 160 p.

MARTINEZ, E. A. et al; As contribuições das representações sociais para a investigação em saúde e enfermagem. Investigación y EducaciónenEnfermería, v. 30, n. 1, p. 101-107, 2012.

MIRLENO, L. M. J; LEITE, R. C. M. Nem só de escola vivo o Ensino de Ciências: Formação científica cidadã no contexto dos museus de ciência. Revista da SBEnBio, Belém, n. 7, p. 5873-5883, out. 2014.

MOREIRA, M. A. Investigação Básica em educação em Ciências: uma visão pessoal. Revista Chilena de Educación Científica, Chile, v. 3, n. 1, p. 10-17, 2004.

MORIGI, V. J. Teoria social e comunicação: representações sociais, produção de sentidos e construção dos imaginários midiáticos. E-Compós, v. 1, p. 1-14, 2004.

MOSCOVICI, S. El psicoanálisis, suimagen y su público. 1 ed. Buenos Aires: Huemul,1979, 363 p.

MOSCOVICI, S. Representações sociais: investigações em psicologia social. 9. ed. Petrópolis: Vozes, 2003. 408 p.

OLIVEIRA, M. 0. Conceito de representações coletivas: uma trajetória da divisão do trabalho às formas elementares. Debates do NER, Porto Alegre, v. 13, n. 22, p. 67-94, 2012.

PAULA, R. M. Representações sociais sobre orgânica de estudantes de ensino médio na cidade de Jundiaí. São Paulo, 2012. 137p. Dissertação (Mestrado em Ensino de Ciências) -Faculdade de Educação, Instituto de Física, Instituto de Química e Instituto de Biociências - Universidade de São Paulo, São Paulo, 2012.

PETTER, C. M. B. Representação Social em Ciências: um estudo preliminar nas séries iniciais do ensino fundamental. Signos, Lajeado, v. 32, n. 1, p. 31-46, 2011.

PICANÇO, A. L. B. A relação entre escola e família: as suas implicações no processo de ensino-aprendizagem. 2012. 152 f. Dissertação (Mestrado em Ciências da Educação) - Escola Superior de Educação João de Deus, Lisboa, 2012.

PINHEIRO-FILHO, F. A noção de representação em Durkheim. Lua Nova [online]. n.61, p.139-155, 2004. Disponível em: http://www.scielo.br/scielo. Acesso em: 20 agosto 2019.

POLÔNIA, A. C; DESSEN, M. A. Em busca de uma compreensão das relações entre família e escola. Psicologia Escolar e Educacional, v. 9, n. 2, p. 303-312, 2005.

PRATTA, E. M. M; SANTOS, M. A. Família e adolescência: a influência do contexto familiar no desenvolvimento psicológico de seus membros. Psicologia em Estudo, Maringá, v. 12, n. 2, p. 247-256, 2007.

SÁ, C. P. Representações Sociais: teoria e pesquisa do núcleo central. Temas em Psicologia, n. 3, p. 19-33, 1996. 
SELINGARDI, G. et al. Representações Sociais de alunos do ensino fundamental I diante 0 instrumento Prova. In: CONGRESSO NACIONAL DE EDUCAÇÃO, n. 12, 2017, Curitiba, Anais [...]. Curitiba: PUC, p. 14771-14782., 2017.

SPINK, M. J. P. The concept of Social Representations in Social Psychology. Cadernos de Saúde Pública, Rio de Janeiro, v. 9, n. 3, p. 300-308, 1993.

TAKAHASHI, B. T. A formação inicial de professores de ciências no estágio supervisionado: compreendendo a identidade docente a partir da teoria das representações sociais e da epistemologia de Ludwik Fleck. 2018. $142 \mathrm{f}$. Tese (Doutorado em Educação para a Ciência e a Matemática) - Centro de Ciências Exatas, Universidade Estadual de Maringá, Maringá. 2018.

TAVEIRA-BISPO, M. A. A importância da participação da família no ensino e aprendizagem escolar de crianças nos anos iniciais do ensino fundamental. Revista Eventos Pedagógicos: articulação universidade e escola nas ações do ensino de Matemática e Ciências. Sinop, v. 6, n. 2, p. 160-169, 2015.

VALA, J. Representações sociais e percepções intergrupais. Análise Social, Lisboa, v. 32, n. 140, p. 7-29, 1997.

VIEIRA, V. et al. Espaços não-formais de ensino e o currículo de ciências. Ciência e Cultura, São Paulo, n. 4, p. 21-23, 2005.

WACHELKE, J. F. R. Índice de centralidade de representações sociais a partir de evocações (INCEV): exemplo de aplicação no estudo da representação social sobre envelhecimento. Psicologia: reflexão e crítica, Porto Alegre, v. 22, n. 1, p. 102-110, 2009.

WACHELKE, J. F. R.; WOLTER, R. Critérios de construção e relato da análise prototípica para representações sociais. Psicologia: teoria e pesquisa. Brasília, v. 27, n. 4, p. 521-526, 2011.

WALSH, F. Processos Normativos da Família: Diversidade e Complexidade. 4 ed. Porto Alegre: Artmed, 2016, 672 p.

ZANCAN, G. T. Educação científica: uma prioridade nacional. São Paulo em perspectiva. São Paulo, v. 14, n. 1, p. 3-7, 2000.

RECEBIDO EM: 08 dez. 2020

CONCLUÍDO EM: 27 abr. 2021 